\title{
Between Scientists, Writers and Artists: Theorising and Critiquing Knowledge-Production at the Interstices between Disciplines
}

\begin{abstract}
Aaron Plasek
Abstract. Stars are Symbols was a collaboration between more than 40 individual writers, poets, artists, and scientists. Each writer/artist conversed with a scientist about the research the scientist was conducting. They then generated new creative work inspired by this process. All the art, creative writing and scientific research was galleried, culminating in an Associated Writing Programmes Conference Off-Site Reading on 7 April 2010. This paper considers some challenging questions that an exhibition like Stars are Symbols engenders. What can we hope to learn about the intersections of science and art by responding to these intersections in discipline-specific modes such as creative writing or fine art? How does one discuss such exhibitions in a precise manner that neither simplifies nor misrepresents ideas in science, nor echoes trite bromides, but helps us recognize new perspectives about the discourses we are considering? Three categories of interdisciplinary work are posited: convergent, radical, and phantasmal. Tentative comments on these questions and others will be offered in the hope of facilitating further discussion.
\end{abstract}

1. How to accommodate contradictory assumptions about knowledge?

Part reading exhibition and part month-long gallery show, the Stars are Symbols project was arranged for writers and fine artists to engage in dialogues with scientists about scientific research. The creative work inspired by these conversations was displayed in a gallery alongside the research artefacts of participating scientists: poems were hung beside derivations of equations modelling supernovae, narratives in the form of flowcharts were suspended alongside diagrams of rocket circuitry, etchings placed abreast of images of high-redshifted galaxies, creative prose beside soil science field notes, and more. My initial intention was to summarize the logistics of how these conversations were facilitated

Aaron Plasek, 'Between Scientists, Writers and Artists: Theorising and Critiquing Knowledge-Production at the Interstices between Disciplines', eds. Nicholas Campion and Rolf Sinclair, Culture and Cosmos, Vol. 16 nos. 1 and 2, 2012, pp. 399-412.

www.CultureAndCosmos.org 
400 Between Scientists, Writers and Artists: Theorising and Critiquing Knowledge-Production at the Interstices between Disciplines

and to consider a few examples of the galleried work that was generated. Discussing these examples would, I hoped, facilitate a critique of Stars are Symbols and enable curators and participants of similar projects to better apprehend 'creative activity markedly different from their own practice and [to help these participants] learn something new about their own creative pursuits by apprehending the pursuits of others'. ${ }^{1}$ I believed such a critique might also identify strategies for engendering new interdisciplinary scholarship and help practitioners of such scholarship to more effectively garner professional recognition within their individual fields.

These initial plans were complicated and challenged by my own lack of theoretical clarity about what form a 'critique' of Stars are Symbols should take: how to critique an endeavour that encompasses participants with radically divergent philosophical assumptions about knowledge and value? One approach for engaging eclecticism is to consider the context in which a work appears and then select methodologies as appropriate. Since Stars are Symbols was instantiated as a gallery show one might restrict the discussion to how science objects are made meaningful when treated as art objects and then ask what significance, if any, this has on our understanding of the arts, the sciences, or the relationship between the two. Such arguments have an extensive precedent. For our purposes Susan Sontag's 'One culture and the new sensibility' and Fairfield Porter's collected critical writings on 'art and science, ${ }^{2}$ from 1966 to 1979 are useful and eloquent examples. Both Sontag and Porter begin their arguments by identifying the motives, philosophical positions, and methodologies of artists and scientists, the explication of which leads to a broader discussion of the functions of 'the sciences' and 'the arts' in terms of the similarities and differences between these broad fields of activity. Sontag and Porter make for fascinating reading not merely because of the extremeness of their positions, but because, to borrow the phrasing of Kenneth Burke, 'Many of the "observations" are but implications of the particular terminology in

1 Aaron Plasek, curator, 'Stars are Symbols' gallery installation, Other Side Arts Gallery, Denver, Colorado, 2010.

2 Susan Sontag, Against Interpretation and Other Essays (New York: Farrar, Straus, and Giroux, 1966), pp. 293-304; Fairfield Porter, Art In Its Own Terms (New York: Taplinger Publishing Company, 1979), pp. 258-282.

Culture and Cosmos 
terms of which the observations are made. ${ }^{3}$ For the scientist or the artist seeking an incisive description of how the sciences and the arts might be related to each other, Sontag and Porter provide lucid examples. Reading these critics side-by-side highlights the similarity in the structure of their arguments despite their seemingly antithetical positions. Sontag identifies similarities between contemporary art and modern science to argue that C. P. Snow's version of the 'Two Cultures' is a false problem born out of a lack of historical knowledge of twentieth-century art. Porter, in his essays 'Art and Knowledge' and 'Technology and Artistic Perception', as well as elsewhere, argues that the arts and the sciences are different enterprises: art is concerned with the 'particular', 'reconciles us to the arbitrary', 'depends on uniqueness', and is 'inexplicable', but science values' $[t]$ he uniformity of nature', a 'similarity' between 'facts', 'prefers to ignore that which there is no hope of reproducing', and is concerned with 'progress away from direct experience and into an insubstantial world of ideas'. ${ }^{4}$ He further argues that this scientific manner of thinking will ' [...]to our very great peril, and even to the peril of our lives, become more and more separated from the inexplicable and immeasurable world of matters of fact'. The sophistication of Sontag's and Porter's writings is difficult to convey in so short a summary. My point is that these critical writings represent the kind of critique I thought to employ to discuss Stars are Symbols. However, since these essays effectively function as manifestos, the manner in which they argue is not suited to discerning new ways of knowing so much as arguing for a particular way of knowing among a number of preexisting alternatives. Such a critique might cause us to be blind to the very 'creative activity markedly different from [our] own practice' I mentioned earlier and of which I had hoped Stars are Symbols would make everyone more cognizant.

I became convinced that, to again borrow Burke's eloquent phrasing, '[W]hat we want is not terms that avoid ambiguity, but terms that clearly reveal the strategic spots at which ambiguities necessarily arise'. ${ }^{6}$ Nor would it be adequate for the purposes of this occasion to discuss individual art pieces, texts, or science artefacts in detail, if such a discussion precluded me from addressing issues of professional

3 Kenneth Burke, Language as Symbolic Action (Berkeley: University of California Press, 1968), p. 46.

4 Porter, Art, pp. 268, 262, 266, and 273.

5 Porter, Art, p. 280.

6 Kenneth Burke, A Grammar of Motives (Berkeley: University of California Press, 1969), p. xviii. 
402 Between Scientists, Writers and Artists: Theorising and Critiquing Knowledge-Production at the Interstices between Disciplines

recognition tied to pursuing interdisciplinary work. How might the work generated from interdisciplinary collaborations be valued and by whom? What possible benefits might it provide for its participants? More to the point, besides the pleasure of conversing on a subject about which one is passionate, what other justifications can we offer for the effort and time required to pursue such interdisciplinary work? If there is a question as to what discipline should house new interdisciplinary work, by what parameters shall we judge the value of the work? And at what point does a collection of questions surrounding an inquiry aggregate into a nascent discipline? These were questions I felt it necessary to explore in order to better understand the activity surrounding Stars are Symbols, and found that interdisciplinary studies scholars frequently considered similar questions when theorizing about models of 'disciplines'. ${ }^{7}$ Disagreements on what constitutes a discipline, as we shall see, ultimately became the basis for a theoretical position sufficiently robust to offer hope of critiquing Stars are Symbols in a manner that did not preclude the possibility of discovering new models of knowledge and knowledge production.

\subsection{Developing a description of disciplines that incorporate the 'strategic spots at which ambiguities necessarily arise'}

The precise parameters we should use to conceptualize the term discipline have been among the most contentious issues in the field of interdisciplinary studies. Dawn Youngblood defines multidisciplinary work as that which happens when 'members of two or more disciplines cooperate' while interdisciplinary work is what happens when new methodologies are developed which 'transcend' or exist outside of current disciplines. ${ }^{8}$ She later

7 Rick Szostak, 'How and Why to Teach Interdisciplinary Research Practice', Journal of Research Practice, Vol. 3, no. 2, article M17 (2007) at http://jrp.icaap.org/index.php/jrp/article/view/92/89 [accessed 10 July 2012]; Paula Viterbo, 'History of science as interdisciplinary education in American colleges: Its origins, advantages, and pitfalls', Journal of Research Practice, Vol. 3, no. 2, article M16 (2007) at

http://jrp.icaap.org/index.php/jrp/article/view/116/96 [accessed 10 July 2011]; Julie Klein, Interdisciplinarity (Detroit: Wayne State University Press, 1990), pp. 14-15, 19-39.

8 Dawn Youngblood, 'Interdisciplinary Studies and the Bridging Disciplines: A Matter of Process', Journal of Research Practice, Vol. 3, no. 2, article M18 (2007): para.1-6, at

Culture and Cosmos 
complicates this picture - correctly, I think-by noting that disciplines have an amorphous quality, arguing that '[disciplines] necessarily overlap, borrow, and encroach upon one another. Within each discipline are sub-disciplines that may behave with as great a sense of separation as exists between separately defined disciplines'. $\quad$ She concludes that our understanding of interdisciplinary work (and disciplinary work by implication) should focus on specific processes rather than a priori definitions. To wit, she notes the domain of geography may be defined as 'the undertaking of describing the earth, ${ }^{10}$ but that this does very little to illuminate what is done in the field or to suggest how this discipline might interact with other disciplines.

Yet Youngblood's model of interdisciplinarity is strange. We can imagine creating a computer animation of Youngblood's disciplines complete with Venn diagrams representing academic disciplines that appear, fragment, and merge in time. Such a hypothetical animated picture suggests little of the underlying relationships between these disciplines, and it's precisely these obscured relationships about which we would like to know something! Instead of generating a set of models using varying definitions of discipline, might we incorporate the fundamental disagreements of what a discipline $i s$ as a feature of our model of interdisciplinarity? I posit that every attempt to develop a methodological model of disciplines and interdisciplinary research is inevitably based on the modeller's answers to the following three questions.

1. To what degree are academic disciplines historically contingent - that is to say, how dependent are the formations of disciplines on cultural, economic, and social forces?

2. To what degree do disciplinary methods determine the existence of facts?

3. To what degree do disciplines change over time and what are the mechanisms that cause new discipline formation?

http://jrp.icaap.org/index.php/jrp/article/view/104/101 [accessed 10 July 2011].

9 Youngblood, 'Bridging Disciplines', para 1-6.

10 Youngblood, 'Bridging Disciplines', para 10.

Culture and Cosmos 
404 Between Scientists, Writers and Artists: Theorising and Critiquing Knowledge-Production at the Interstices between Disciplines

Arguing that disciplinary methods construct facts (question 2) is not to say there are no better or worse ways to do something, nor is it to imply that there is no physical reality. Instead this question is intended to bring explicit attention to the degree to which the methods employed highlight certain 'facts' while hiding other kinds of facts that would have been apparent had we used a different set of methods. Readers interested in exploring the appropriateness of these questions as fundamental disagreements within the discipline of interdisciplinary studies may find edification in comparing the arguments of William Newell's to those of Richard Carp's as presented in their respective essays 'A Theory of Interdisciplinary Studies' and 'Integrative Praxes: Learning from Multiple Knowledge Formations'; however, space considerations do not permit a detailed discussion of those two interesting articles here. ${ }^{11}$ Instead I wish to pose tentative terms robust enough to discuss the entire range of potential answers to the three questions above. This allows us to critique Stars are Symbols in a manner more sensitive to differing (and even opposing) conceptions of knowledge and work, denote the limitations of the project as it was then conceived and executed, and help us envision ways in which future similar projects can increase opportunities for meaningful interdisciplinary collaboration.

\subsection{Three terms for describing interdisciplinary work}

First, let us define convergent interdisciplinary work to be collaborations between individuals across disciplines that use a set of commensurable methods that are already shared between disciplines. I use the term commensurable methods to connote methods, assumptions, beliefs, theories and so forth that produce facts that can be evaluated using similar kinds of (repeatable within the context of the discipline) tests or procedures. One example of convergent interdisciplinary work is the collaboration of computer

11 William H. Newell, 'A Theory of Interdisciplinary Studies', Issues in Integrative Studies, Vol. 19 (2001), pp. 1-25, at http://www.units.muohio.edu/aisorg/pubs/issues/19 Newell.pdf [accessed 10 July 2011]; Richard M. Carp, 'Integrative Praxes: Learning from Multiple Knowledge Formations', Issues in Integrative Studies, Vol. 19 (2001), pp. 71121, at http://www.units.muohio.edu/aisorg/pubs/issues/toc voll9.shtml [accessed 10 July 2011].

Culture and Cosmos 
scientists and astrophysicists to numerically model and visualize supernovae explosions at the ASC Flash Center at the University of Chicago. ${ }^{12}$ Some kinds of complexity research being conducted at the Santa $\mathrm{Fe}$ Institute are another useful example of convergent collaborations. ${ }^{13}$ An important social consequence of tying the definition of convergent interdisciplinary work to the use of commensurable methods is that the work an individual does in such a collaboration is recognized by his or her academic department of origin as 'legitimate' professional work and is rewarded with institutional benefits. Since this work can be judged (either accurately or poorly) with existing disciplinary methods, convergent interdisciplinary work is frequently subsumed by preestablished departmental disciplines.

In this way, convergent interdisciplinary work tends to consolidate the power of existing academic departments, as has been suggested explicitly by Dawn Youngblood and implicitly by William Newel when he writes, '[I]nterdisciplinary integration is driven by the tension between disciplinary insights and phenomenological pattern'. ${ }^{14,15}$ Rick Szostak also conceptualizes interdisciplinary research as both convergent and as strengthening existing disciplines in his argument that disciplinary and interdisciplinary are symbiotic terms, noting ' $[\ldots \mathrm{A}] 11$ interdisciplinary research needs to grapple with questions of how and why to integrate the insights of different disciplines'. ${ }^{16}$

Second, let us define radical interdisciplinary work as fundamentally different from (but not mutually exclusive of) convergent collaboration. While convergent collaboration is marked by shared (commensurable) methods between disciplines, radical interdisciplinary collaboration employs methods from across

12 Flash Center for Computational Physics, University of Chicago, 'Organization of the Flash Center' at

http://flash.uchicago.edu/site/about/organization/ [accessed 20 July 2011].

13 Dan Dillion, 'A Review of the Santa Fe Institute: Institutional and Individual Qualities of Expert Interdisciplinary Work', Interdisciplinary Studies Project: Project Zero, Harvard Graduate School of Education (2001), at http://pzweb.harvard.edu/interdisciplinary/pdf/Dillon SantaFe 2001.pdf [accessed 20 July 2011].

14 Youngblood, 'Bridging Disciplines', para. 6.

15 Newell, 'Theory of Interdisciplinary', p. 20.

16 Szostak, 'How and Why', para. 2 in sec. 3. 
406 Between Scientists, Writers and Artists: Theorising and Critiquing Knowledge-Production at the Interstices between Disciplines

different disciplines that are incommensurable (that is, there is no obvious way to compare the 'facts' generated by one method with the 'facts' generated by a method incommensurable to the first). Radical work necessarily produces new methods which find ways to link earlier methods and facts that were previously incommensurable; it does this, in part, by changing the contexts and meanings of the original terms. As one might infer from the previous discussion of convergent interdisciplinary collaboration, radical interdisciplinary work may not garner institutional benefits because it may be unrecognisable as professional work within the existing disciplines. Individuals who would engage in radical interdisciplinary work are pragmatically discouraged (though not disallowed) from doing so by existing (academic) disciplines. Radical interdisciplinary work may be subversive to existing disciplines as Carp has argued, noting, '[...] I understand 'interdisciplinarity' as a search not only for new 'knowledge' but also new ways to know and of new 'things' to be known, including new social relations that generate and validate knowledge[.] ${ }^{17}$ Such radical collaborations may also spawn nascent disciplines, though that interesting consequence will not be directly discussed in this paper.

Linking radical and convergent interdisciplinary work to the discipline-specific methodologies suggests a third kind of collaboration I shall call phantasmal and that appears to be interdisciplinary, but in which the specific methodologies of different disciplines remain effectively isolated from one another. The participants in phantasmal interdisciplinary collaborations may be influenced by specific disciplinary ideas or models outside of their own discipline, but the methods these participants use to produce new work remain unchanged by the collaboration. Likewise, the criteria for judgment of this phantasmal work remain completely within the discipline within which it was produced. Of the three terms I define here, phantasmal work is the most problematic to define precisely and highlights the limitations of using a methodological framework to discuss disciplines. Phantasmal work may adopt rhetoric that sounds like it is interdisciplinary by borrowing terms from other disciplines without having an understanding of their meaning in their original context

17 Carp, 'Integrative Praxes', p. 85.

Culture and Cosmos 
in an attempt to mimic convergent work. Phantasmal work may also mirror radical work in the sense that incommensurable methods seem to be used to generate new methods; but such 'new methods' are ultimately a superficial repackaging of existing disciplinary methods.

Having taken care to identify terms that highlight several ways disciplines can interact while also not presuming any correctness about what knowledge must be, it will behove us to examine the execution of Stars are Symbols in more detail before proceeding with our critique.

\section{A brief compendium of procedures, participants and logistical} details for the 2010 Stars are Symbols

The gallery show component of the Stars are Symbols project exhibited the work of 40 individuals during April 2010 at the Other Side Arts Gallery in Denver, Colorado. A complete list of the participants and the fields in which they provided work for Stars are Symbols is detailed in Table 1. The gallery component of Stars Are Symbols was bookended by two live performances, also held at the Other Side Arts. The opening exhibition on 7 April 2010 featured readings by fourteen Stars Are Symbols writers, and was purposely chosen to coincide with the start of the 2010 Associate of Writers \& Writing Programs (AWP) Conference held in Denver that year. The closing exhibition of the gallery show featured a musical performance, influenced by the music that Johannes Kepler saw in the orbits of the planets and that he detailed in Harmonices Mundi (Harmony of the Planets). Approximately 500 people attended the gallery show, including over 150 people who attended the opening and closing exhibitions.

Face-to-face conversations between participants were often unfeasible because of geographical distances. The 2010 Stars are Symbols had participants from thirteen states and three different countries. Pairings between scientists, writers, and artists were facilitated by myself via emailed introductions and were usually conducted via Skype or phone at a time arranged between the scientist and the writer/artist. Scientist-Artist/Writer pairs were instructed to speak with each other for no less than fifteen minutes. Most conversations were longer and a few exceeded three hours. The dialogues of the project were 
408 Between Scientists, Writers and Artists: Theorising and Critiquing Knowledge-Production at the Interstices between Disciplines

\begin{tabular}{|c|c|}
\hline SCIENTISTS & WRITERS / ARTISTS \\
\hline \multirow{2}{*}{ Azure Avery, physics } & Janelle Welsh, poetry ${ }^{\alpha}$ \\
\hline & $\begin{array}{l}\text { Jonathan Wiley, print mak- } \\
\text { ing }{ }^{\beta}\end{array}$ \\
\hline \multirow{2}{*}{ Alan Calder, astrophysics } & Adi Nott, painting \\
\hline & Jenifer Park, poetry ${ }^{\alpha}$ \\
\hline \multirow{2}{*}{ John Debes, astronomy } & Emily Anderson, poetry \\
\hline & Susan Tepper, poetry ${ }^{\alpha}$ \\
\hline Christian Fritz, physics & Adam Jameson, fiction \\
\hline Derek Lamb, astronomy & David Bowen, fiction ${ }^{\alpha}$ \\
\hline \multirow{2}{*}{ Jamie Lomax, physics } & Caryl Pagel, poetry \\
\hline & Jake Adam York, poetry ${ }^{\alpha}$ \\
\hline \multirow{2}{*}{ M. Leila Mays, astronomy } & Charles Malone, poetry ${ }^{\alpha}$ \\
\hline & Dylan Scholinski, painting \\
\hline Charles Nelson, astronomy & Mary Hamilton, fiction \\
\hline \multirow{3}{*}{ Athanasios Petridis, physics } & Michelle Taransky, poetry \\
\hline & Carina Gia Farrero, poetry \\
\hline & Rico Moore, poetry \\
\hline $\begin{array}{l}\text { Jonathan Sowder, soil sci- } \\
\text { ence }\end{array}$ & Nicolette Bond, poetry ${ }^{\alpha}$ \\
\hline \multirow{3}{*}{ Mark Piper, engineering } & Daniel Borzutsky, fiction ${ }^{\alpha}$ \\
\hline & $\begin{array}{l}\text { Shawn Huelle, book art / fic- } \\
\text { tion }^{\alpha}\end{array}$ \\
\hline & $\begin{array}{l}\text { Brendan Healy, visual art / } \\
\text { fiction }\end{array}$ \\
\hline \multirow{2}{*}{ Sarah Ragan, astronomy } & John Gallaher, poetry ${ }^{\alpha}$ \\
\hline & Adam Peterson, fiction ${ }^{\alpha}$ \\
\hline \multirow{4}{*}{ Greg Ver Steeg, physics } & Matthew Cooperman, poetry ${ }^{\alpha}$ \\
\hline & Aby Kaupang, poetry ${ }^{\alpha}$ \\
\hline & Bin Ramke, poetry \\
\hline & G. C. Waldrep, poetry \\
\hline $\begin{array}{l}\text { Kepler's Harmony of the } \\
\text { Planets }\end{array}$ & Daniel Christensen, music ${ }^{\beta}$ \\
\hline
\end{tabular}

TABLE 1: Pairings in the 2010 Stars are Symbols gallery show. The $\alpha$ and $\beta$ superscripts denote individuals performing in the 7 April and 30 April exhibitions, respectively. Most scientists participated in multiple pairings. 
formally structured to be one-sided: the scientist discussed his or her work, but there were no formal requirements for the artist or poet to do the same. Second, the project accepted contributions from participants in a comparably one-sided fashion: the artists and writers were required to produce new work while the scientists were directed to use existing results.

\section{Critique of Stars are Symbols and suggestions for future collaboration}

3.1. Stars are Symbols produced phantasmal collaborations and little else. It is not obvious what the ideal venue may be for radical collaborations until after they are completed.

The 2010 Stars are Symbols, at best, was a phantasmal interdisciplinary collaboration: the writers and artists - inspired by some ideas presented by the scientists - proceeded to generate new work in fundamentally the same way they had in the past, but borrowing ideas and jargon taken from the scientists; likewise, the scientists continued to conduct their professional work using the same methods they had employed prior to the project. Regardless of how exciting, interesting, entertaining, or profound the work from phantasmal interdisciplinary collaborations are to the curators and participants of such projects, these collaborations will always be inherently unsatisfying from a theoretical perspective because the methodologies of the participants remain fundamentally unchanged. Future Stars are Symbols should enable more convergent and/or radical collaborations.

The exact form radical interdisciplinary work will take is difficult to anticipate. As previously discussed, it is unlikely that such work will be recognized in existing departmental disciplines, so the appropriate venue for this new work may not be obvious. The ideal future Stars are Symbols would inhabit a variety of different venues chosen in response to the work generated. Practical considerations make this difficult. While not being able to accommodate every work, the ability of a website to quickly switch between a variety of graphical modes of presentation allows curators more flexibility and also allows the interdisciplinary work a far larger potential audience not bound by geographical or temporal constraints. 
410 Between Scientists, Writers and Artists: Theorising and Critiquing Knowledge-Production at the Interstices between Disciplines

\subsection{Stars are Symbols did not provide professional recognition for all participants}

For writers and artists the 2010 Stars are Symbols afforded professional rewards. The creative works generated in Stars are Symbols could be published elsewhere (and frequently have been), and their colleagues recognized participation in Stars are Symbols as work in the field. For scientists participating in Stars are Symbols, neither the dialogues, nor the gallery show, nor the exhibitions garnered overt disciplinary rewards (though some scientists might argue that their work in the gallery show contributed to greater public awareness of science in general as well as greater awareness of their particular research). The existence (or lack) of professional rewards places practical and often detrimental constraints on interdisciplinary projects like Stars are Symbols.

The curatorial decision, for example, of asking scientists to submit pre-existing work minimized the time commitment from them and allowed more interested scientists to participate in the project, but at the high cost of discouraging convergent or radical interdisciplinary collaboration. If we wish future interdisciplinary projects like Stars are Symbols to elicit more significant contributions of original interdisciplinary work that is convergent and/or radical from scientists, curators must find ways to increase the disciplinary rewards for participating. Ensuring convergent interdisciplinary collaborations for writers, artists, and scientists would provide additional professional motivation to invest the attention, energy, and effort that radical collaboration frequently requires.

3.3. Some disciplines recognise a greater variety of activities and products as professional work than other disciplines. For example, artists and writers have a greater range of products that are considered 'professional' work in their fields than do physical scientists. Projects that seek to engender convergent or radical collaboration must address the variability of professional work for each discipline involved.

Fine artists and creative writers are able to use projects like Stars are Symbols to create convergent interdisciplinary work because their disciplines recognize a broad range of products as professional work. Scientists have a much narrower range of products recognized as professional work and thus it may appear difficult for 
their participation in projects like Stars are Symbols to ever be convergent. While a rigorous discussion of how convergent participation might be achieved for scientists requires its own paper, splitting convergent collaborations into those that serve a public function of recognition and those that serve a pragmatic function within the day-to-day activities of research leads us to two possible suggestions.

First, a scientist may argue that the mandate of many professional scientific funding organizations like the National Science Foundation is met through their participation in radical interdisciplinary projects like Stars are Symbols that '[]infuse learning with the excitement of discovery, and assure that the findings and methods of research are communicated in a broad context and to a large audience'. ${ }^{18}$

Second, regarding the potential of radical collaborations to help scientists conduct research, we may borrow an argument posited by Paul Feyerabend in Against Method: using 'counterrules' to produce hypotheses that contradict existing accepted scientific theories enables us to develop 'important formal properties of an [accepted] theory ${ }^{19}$ by the contrasts provided by the counterfactual theories. A scientist thus does not merely proceed by considering experimental evidence against a theory but, as Feyerabend writes: ' $[\mathrm{H}] \mathrm{e}$ must adopt a pluralistic methodology. $\mathrm{He}$ must compare ideas with other ideas rather than with "experience" and he must try to improve rather than discard the views that have failed in competition [.... $]^{20}$

Many subfields in physics are littered with examples of scientists that proceeded at times by employing ideas that contradicted existing physical theories. A list of examples that benefited from counter-inductive hypotheses might include: the decision to treat time as a variable in special relativity, the Copenhagen interpretation of quantum mechanics and the inability to locate a medium through which electromagnetic waves propagate. And no discipline has held a monopoly on ideas that were useful to the advancement of scientific research. If theories

18 National Science Foundation Graduate Research Program, 'Review Criteria' at http://www.nsfgrfp.org/how_to_apply/review_criteria [accessed 25 July 2011].

19 Paul Feyerabend, Against Method (New York: Verso, 1988), pp. 20 - 21.

20 Feyerabend, Against Method, pp. 20 - 21. 
412 Between Scientists, Writers and Artists: Theorising and Critiquing Knowledge-Production at the Interstices between Disciplines

counterfactual to the sciences and the organizational patterns of other disciplines can aid the thinking of scientists, there is no reason that projects like Stars are Symbols cannot provide a scientist with insights into his or her research.

\section{Acknowledgments}

My grateful acknowledgement to Metropolitan State University of Denver for offsetting some of the expenses related to attending INSAP VII. My sincere thanks as well to Emilie Vergé, Ulisses Barres de Almeida, and Berco Wilsenach who made INSAP VII extremely enjoyable and whose conversations challenged and stimulated me. Gratitude is also due to Greg Ver Steeg, Farah Godrej, Matthew Plasek, and Sam Robinson for providing a summer in Los Angeles conducive to writing these proceedings. 